\title{
Synthetic Aperture Focusing Applied to Imaging Using a Rotating Single Element Transducer
}

Kortbek, Jacob; Jensen, Jørgen Arendt; Gammelmark, Kim Løkke

Published in:

IEEE Ultrasonics Symposium, 2007

Link to article, DOI:

10.1109/ULTSYM.2007.378

Publication date:

2007

Document Version

Publisher's PDF, also known as Version of record

Link back to DTU Orbit

Citation (APA):

Kortbek, J., Jensen, J. A., \& Gammelmark, K. L. (2007). Synthetic Aperture Focusing Applied to Imaging Using a Rotating Single Element Transducer. In IEEE Ultrasonics Symposium, 2007 (pp. 1504-1507). IEEE.

https://doi.org/10.1109/ULTSYM.2007.378

\section{General rights}

Copyright and moral rights for the publications made accessible in the public portal are retained by the authors and/or other copyright owners and it is a condition of accessing publications that users recognise and abide by the legal requirements associated with these rights.

- Users may download and print one copy of any publication from the public portal for the purpose of private study or research.

- You may not further distribute the material or use it for any profit-making activity or commercial gain

- You may freely distribute the URL identifying the publication in the public portal 


\title{
Synthetic aperture focusing applied to imaging using a rotating single element transducer
}

\author{
Jacob Kortbek*†, Jørgen Arendt Jensen* and Kim Løkke Gammelmark ${ }^{\dagger}$ \\ ${ }^{*}$ Center for Fast Ultrasound Imaging, Ørsted•DTU, \\ Bldg. 348, Technical University of Denmark, DK-2800 Lyngby, Denmark \\ ${ }^{\dagger}$ B-K Medical, Mileparken 34, DK-2730 Herlev, Denmark.
}

\begin{abstract}
This paper applies the concept of virtual sources and mono-static synthetic aperture focusing (SAF) to 2-dimensional imaging with a single rotating mechanically focused concave element with the objective of improving lateral resolution and signal-to-noise ratio (SNR). The geometrical focal point of the concave element can be considered as a point source emitting a spherical wave in a limited angular region. The SAF can be formulated as creating a high resolution line as a sum over low resolution lines (LRL). A LRL is the contribution from a single emission. Simulation in Field II is based on moving the concave element of radius $2.5 \mathrm{~mm}$ along a circle of radius $10 \mathrm{~mm}$. Elements with different concave curvatures are used to obtain geometrical focusing depths at $10 \mathrm{~mm}, 15 \mathrm{~mm}$, and $20 \mathrm{~mm}$. Point targets in the range from $5 \mathrm{~mm}$ to $65 \mathrm{~mm}$ are used as image objects. The high resolution images (HRI) are shown and the radial and angular resolution are extracted at $-6 \mathrm{~dB}$ and $-40 \mathrm{~dB}$. The performance of the setup with a VS at $20 \mathrm{~mm}$ is superior to the other setups. Due to the rotation, the synthesized aperture only experiences a moderate expansion, which is not sufficient to reduce the extent of the wide point spread function of a single emission. The effect of SAF with focal depth at 20 $\mathrm{mm}$ is negligible, caused by the small number of LRL applied. The great profit of the SAF is the increase in SNR. For the setup with focal depth at $20 \mathrm{~mm}$ the SAF SNR gain is $11 \mathrm{~dB}$. The SNR gain of a setup with a VS at radius $10 \mathrm{~mm}$ or $15 \mathrm{~mm}$ over conventional imaging with a VS at $20 \mathrm{~mm}$, is also $11 \mathrm{~dB}$.
\end{abstract}

\section{INTRODUCTION}

Inspired by synthetic aperture radar techniques, synthetic aperture ultrasound imaging has been investigated thoroughly for many years. In synthetic transmit aperture (STA) imaging a single element is used in transmit to create a spherical wave that propagates in all directions. In receive a multielement aperture is applied. The backscattered signals come from all imaging points and can be used to construct a lowresolution image (LRI), hence an image constructed from a single emission. Several emissions from single elements across the aperture can be used to create several LRI's which can be added into a high-resolution image (HRI). In the monostatic approach [1] the same element serves as a transmitter and a receiver. Variations of synthetic transmit aperture and sparse synthetic aperture techniques has been investigated with improvements in both frame rate, penetration, and lateral resolution. A synthetic aperture technique suitable for a hand held system using a multi-element transmit and receive aperture was described by Karaman et al. [2]. Lockwood and Hazard described a sparse synthetic aperture beamforming technique for three-dimensional ultrasound imaging using a few transmit pulses for each image [3], [4]. A synthetic aperture method for a circular aperture was investigated by O'Donnell and Thomas [5]. The concept of using the transmit focal point as a virtual aperture was introduced by Passmann and Ermert [6]. Virtual sources in synthetic aperture focusing (SAF) was further investigated by Frazier and OBrien [7], Nikolov and Jensen [8], [9], and Bae and Jeong [10].

This paper applies the concept of virtual sources and synthetic aperture techniques to 2-dimensional imaging with a single rotating mechanically focused concave element. Such an imaging system can e.g. be found in an anorectal ultrasound transducer. With such a transducer emission and reception are done while the transducer element continuously rotates and the received RF signals are stored. It is proposed to apply monostatic synthetic aperture focusing (SAF) on these data with the objective of improving lateral resolution and signal-to-noise ratio (SNR). This is investigated using simulations in Field II [11], [12] and SAF is carried out using the Beamformation Toolbox, BFT2 [13]. Images of point targets are created and the radial and angular resolution in the polar coordinate system is extracted. The potential improvement in SNR is also evaluated. The SAF method is described in Section II. The simulation setup and simulation results are found in Section III, and the investigation is concluded in Section IV.

\section{METHOD}

In the mono-static case of linear array SAF, a single element is used in both transmit and receive. The transmitting element can be considered a point source emitting a spherical wave. The wave traverses all image points and an entire LRI can be formed after each emission. Several emissions from across the aperture can be used to construct a HRI. The HRI has a better resolution than the LRI, since the multiple emissions synthesize an effective aperture of a certain lateral width.

This focusing scheme can not directly be applied to imaging with a rotating mechanically focused element. To begin with the lateral translation of the transmit source is smaller for the rotating system than for the linear array making the synthesized width of the aperture smaller. Secondly the concave rotating element can not be considered a point source because of the physical extent of the element and the geometrical focusing due to the concave shape. Instead the geometrical 
focal point can be considered as a point source emitting a spherical wave in a limited angular region. The focal depth of the transducer element or more exact the focusing $F_{\#}$ determines the opening angle, hence the width of the wave field and the point spread function (PSF). An illustration of propagating waves from 3 different emissions is shown in Fig. 1 for a transducer element with a focal depth of $10 \mathrm{~mm}$.

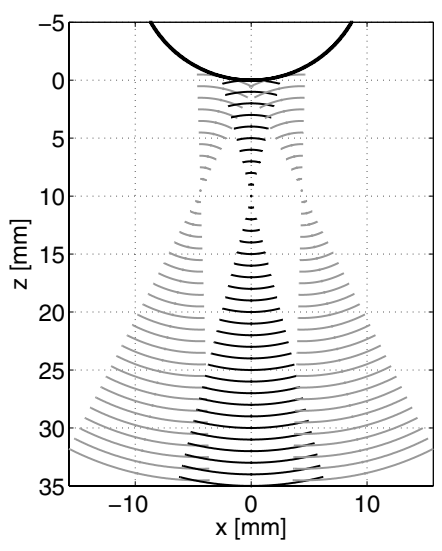

Fig. 1. An illustration of propagating waves from 3 different emissions for a transducer element with a focal depth of $10 \mathrm{~mm}$.

A LRI of the entire imaging area can not be formed after each emission due to the spatially limited wave propagation. Instead, for each image point in a high resolution image line (HRL) it must be determined which emissions that have a wave field that encompasses the image point. These emissions contribute to the HRL, and samples from each of them are selected according to the SAF delays, and added together. In other words a single HRL is composed of the sum of a number of low resolution lines (LRL). A single LRL is, thus, the contribution from a given emission to a HRL. The term LRL is used for an RF-line which have been processed using SAF and which contains at least one non-zero sample which carries information of one of the image points of the HRL.

The SAF can be formulated as a sum over LRL. A single sample of the HRL, representing the image point at the location $\vec{r}_{i p}$ with angular coordinate $\phi$ and radial coordinate $r$ becomes

$$
h(\phi, r)=\sum_{\theta} \mathcal{A}(\theta, r) l_{\theta}(r),
$$

where $h(\phi, r)$ is the HRL sample, and $l_{\theta}(r)$ is the LRL sample from the emission with propagation direction $\theta$. Both with radial coordinate $r$. The variable $\mathcal{A}$ is an apodization function which controls the weighting of the contribution from each of the LRL. It is a function a the radial coordinate, since the number of contributing emissions increases with range. The synthesized aperture increases with range resulting in a less range dependent lateral resolution. The LRL can be formulated using the RF-line, $s_{\theta}\left(r^{\prime}\right)$

$$
l_{\theta}(r)=s_{\theta}\left(r^{\prime}\right) .
$$

$r^{\prime}$ is the radial coordinate at which to select a sample from the RF-line and is not the same radial coordinate as in the HRL. $r^{\prime}$ can be found from the distance function, $r^{\prime}=d\left(\vec{r}_{v_{\theta}}, \vec{r}_{i p}\right)$ which calculates the transmit-receive time-of-flight for the $\mathrm{SAF}$, and thus the sample index for the RF-line, $s_{\theta}\left(r^{\prime}\right)$. The transducer element at the position $\vec{r}_{t_{\theta}}$ is physically focused at the VS at the position $\vec{r}_{v_{\theta}}$ with a focal distance of $d_{v}$ from the element. The element is focused in both transmit and receive and the distance function becomes a sum of transmit and receive travel paths

$$
\begin{array}{rrr}
d\left(\vec{r}_{v_{\theta}}, \vec{r}_{i p}\right)= & & \left|\vec{r}_{v_{\theta}}-\vec{r}_{t_{\theta}}\right| \pm\left|\vec{r}_{i p}-\vec{r}_{v_{\theta}}\right| \\
& \pm\left|\vec{r}_{v_{\theta}}-\vec{r}_{i p}\right|+\left|\vec{r}_{t_{\theta}}-\vec{r}_{v_{\theta}}\right| \\
= & 2\left|\vec{r}_{v_{\theta}}-\vec{r}_{t_{\theta}}\right| \pm 2\left|\vec{r}_{i p}-\vec{r}_{v_{\theta}}\right| \\
= & 2 d_{v} \pm 2\left|\vec{r}_{i p}-\vec{r}_{v_{\theta}}\right|
\end{array}
$$

The \pm in (3) refer to whether the image point is above or below the VS. A single sample of the HRL can thus be formulated using (1) and (2)

$$
h(\phi, r)=\sum_{\theta} \mathcal{A}(\theta, r) s_{\theta}\left(d\left(\vec{r}_{v_{\theta}}, \vec{r}_{i p}\right)\right) .
$$

A single element of limited extent can be considered as a wave source emitting a spherical wave, which will cover the entire image area. With complete overlap between the covered image areas all emission can be utilized to create a HRI. Having a physically focused transducer element the VS is conceived as the wave source. With a transducer element of fixed size the focal depth determines the opening angle, $\alpha$ and the confined image area covered by the wave field. The consequence is that not all emissions can be utilized to create a HRI. Having a rotating element instead of a translating element even further limits the number of applicable emissions. From geometrical observation the opening angle can be expressed as $\alpha=\arctan \frac{1}{2 F_{\#}}$, which is a valid approximation [14]. The confined image area covered by the wave field is, thus, a function of the $F_{\#}$. With a higher $F_{\#}$ the point spread function becomes narrower improving the resolution for the single RFline. This portrays a conflict between using a low $F_{\#}$ and adding a large number of LRL, and using a high $F_{\#}$ and reducing the number of applied LRL. Performance results for these different configurations are presented in Section III.

\section{Simulation Results}

The SAF scheme is investigated using Field II [11], [12]. The simulations are based on moving a single concave element of radius $2.5 \mathrm{~mm}$ along a circle of radius $10 \mathrm{~mm}$. Elements with different concave curvatures are used to obtain geometrical focusing depths at $10 \mathrm{~mm}, 15 \mathrm{~mm}$, and $20 \mathrm{~mm}$. The scattering media consists of point targets (PT) in the range from $5 \mathrm{~mm}$ to $65 \mathrm{~mm}$ with a distance of $10 \mathrm{~mm}$. The transducer element is rotated with a fixed angle of $0.63^{\circ}$ corresponding to moving the element $\lambda / 2$ between each acquisition. It is assumed that the transducer element is not moving during emission and reception. The $7 \mathrm{MHz}$ pulse, and the one-way impulse response are weighted single-cycle sinusoids.

The envelope HRI's are shown in this section. The radial and angular resolution are extracted at $-6 \mathrm{~dB}$ and $-40 \mathrm{~dB}$. For 
each PT the resolution is calculated as the width at which the envelope amplitude has decreased to the specified level relative to the maximum level at that given PT. For the lateral resolution the envelope amplitude is taken as the maximum amplitude within a radial range of $\pm 3 \mathrm{~mm}$ around the PT. Likewise for the radial resolution the envelope amplitude is taken as the maximum amplitude across the entire angular range of the image.

The variable $\mathcal{A}$ from (4) controls the weighting of the contribution from each of the LRL and is a function a the radial coordinate. The actual number of contributing LRL is shown in Fig. 2 for the transducer elements with focusing depths at $10 \mathrm{~mm}, 15 \mathrm{~mm}$, and $20 \mathrm{~mm}$. As the focal depth increases, and the opening angle decreases, the number of contributing LRL decreases.

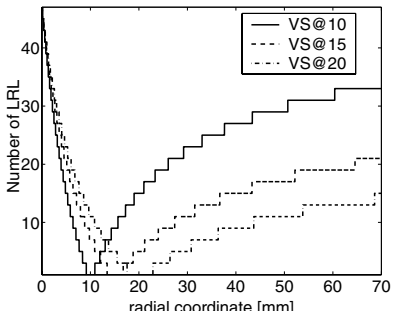

Fig. 2. The number of contributing LRL for the transducer elements with focusing depths at $10 \mathrm{~mm}, 15 \mathrm{~mm}$, and $20 \mathrm{~mm}$.

Images with different setups are shown in Fig. 3. In the first three images the focusing depths of the element are $10 \mathrm{~mm}$, $15 \mathrm{~mm}$, and $20 \mathrm{~mm}$, respectively and SAF has been applied. In the last image the element has a focusing depths of $20 \mathrm{~mm}$ and SAF is not applied. The differences between the images are better demonstrated in Fig. 4 where the radial and angular resolution are extracted. The performance of the setup with a VS at $20 \mathrm{~mm}$ is superior to the other setups. This reflects the conflict stated earlier between using a low $F_{\#}$ and adding a large number of LRL, and using a high $F_{\#}$ and reducing the number of LRL. Due to the rotation the synthesized aperture only experiences a moderate expansion even for the setup with a focal depth of $10 \mathrm{~mm}$. The modest expansion is not sufficient to reduce the extent of the wide point spread function of a single emission. At least not to a level which is competitive to the relatively narrow PSF of the setup with focal depth at $20 \mathrm{~mm}$. The effect of SAF with focal depth at $20 \mathrm{~mm}$ is negligible. This is also caused by the small number of LRL. Only $15 \mathrm{LRL}$ are used at a range of $70 \mathrm{~mm}$ as shown in Fig. 2.

The SAF technique rely on phase coherent addition of the LRL. The LRL's composing the center HRL around the PT at $35 \mathrm{~mm}$ are plotted in Fig. 5. The setup is with an element with focal depth of $10 \mathrm{~mm}$. It is apparent that the LRL's are completely phase aligned and add up constructively.

Provided that the image object does not move in between acquisitions and assuming uncorrelated electronic noise at the receivers the SNR improvement using SAF is

$$
\Delta S N R(r)=10 \log _{10}\left(N_{r_{v}}(r)\right) \text {. }
$$
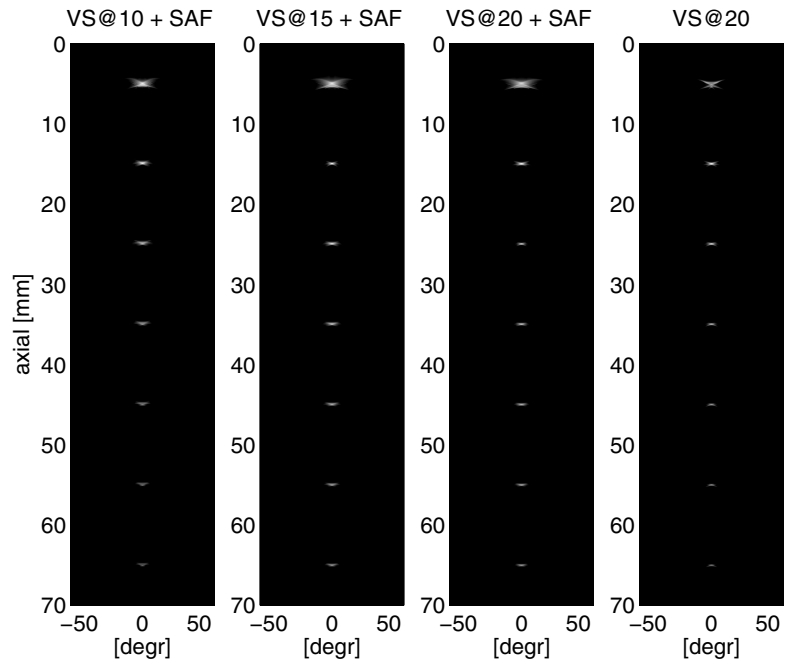

Fig. 3. Images from 4 different setups. In the first three images the focusing depths of the elements are $10 \mathrm{~mm}, 15 \mathrm{~mm}$, and $20 \mathrm{~mm}$ respectively and SAF has been applied. In the last image the element has a focusing depths of 20 $\mathrm{mm}$ and SAF is not applied. Dynamic range is $60 \mathrm{~dB}$.
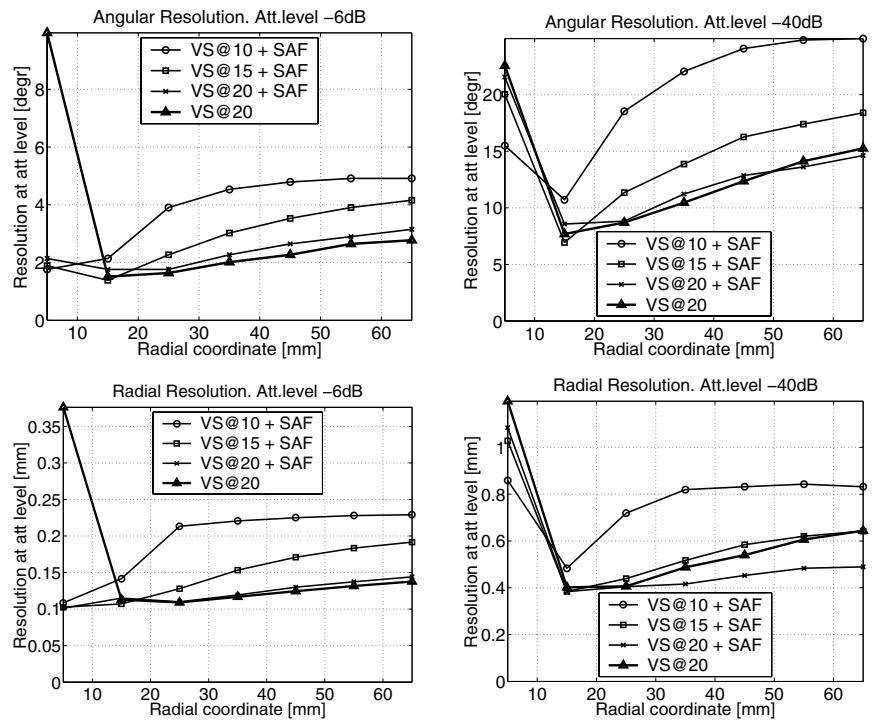

Fig. 4. Radial resolution (bottom) and angular resolution (top) at $-6 \mathrm{~dB}$ (left) and $-40 \mathrm{~dB}$ (right)

$N_{r_{v}}(r)$ is the number of emissions which contributes to the HRL for a transducer element with a VS at radius $r_{v}$ as shown in Fig. 2. With a VS at $20 \mathrm{~mm} N_{r_{v}}(r)=13$ at radius, $r=60$ $\mathrm{mm}$. This yields a SAF SNR improvement of $\Delta S N R=11$ dB. With a lower $F_{\#}$ the number of contributing emissions increases, but the width of the wave field does also increase. Provided that the transmitted power is equally distributed over the width of the field, the SAF SNR improvement of a setup with a VS at radius $r_{v}$ over conventional imaging with a VS at $20 \mathrm{~mm}$ can be expressed as

$$
\Delta S N R_{r_{v}}(r)=10 \log _{10}\left(N_{r_{v}}(r) \frac{W_{r_{20}}(r)}{W_{r_{v}}(r)}\right) .
$$

$W_{r_{20}}$ and $W_{r_{v}}$ are the $-6 \mathrm{~dB}$ widths of the wave fields 

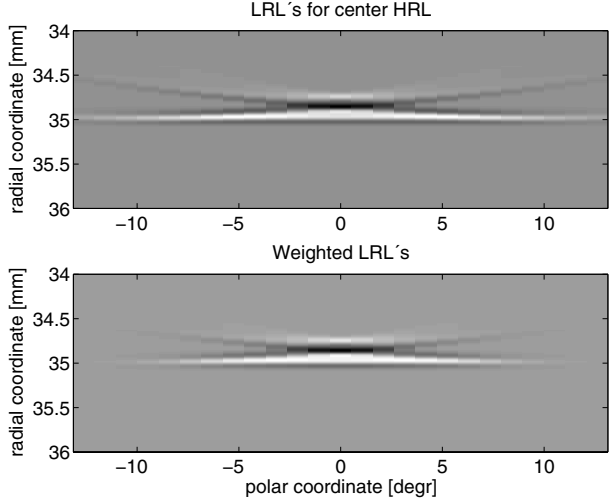

Fig. 5. A single HRL is composed of the sum of a number of low resolution lines (LRL). Here the LRL's composing the center HRL around the PT at 35 $\mathrm{mm}$ are shown. The setup is with an with focal depth of $10 \mathrm{~mm}$. Without apodization (top) and with Hamming apodization (bottom).

measured in whole wave lengths for the elements with VS's at radius $20 \mathrm{~mm}$ and at radius $r_{v}$, respectively. The widths are calculated using the opening angle, $\alpha$. The width-ratio and the SNR improvement are shown in Fig. 6 for elements with VS's at radius $r_{v}=10 \mathrm{~mm}, r_{v}=15 \mathrm{~mm}$, and $r_{v}=20 \mathrm{~mm}$. At greater range the width-ratio is 2.5 , and 1.5 with elements having VS's at radius $r_{v}=10 \mathrm{~mm}, r_{v}=15 \mathrm{~mm}$ respectively, while $N_{r_{v}}(r)$ is 33 and 21 . This results in an improvement in SNR of $11 \mathrm{~dB}$ in both cases just as the improvement of using SAF on the setup with the VS at radius $20 \mathrm{~mm}$. The SNR improvement can be converted directly to an increase in penetration or an increase in emitted frequency yielding a higher resolution.
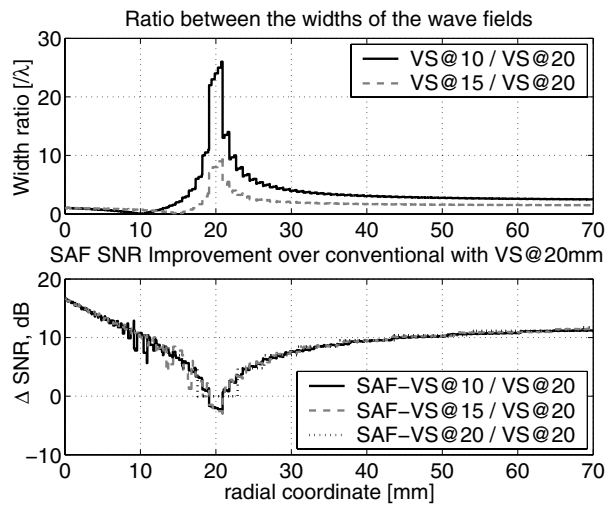

Fig. 6. The ratio between the width of different wave fields (top) and SAF SNR improvement over conventional imaging with a VS at radius $20 \mathrm{~mm}$ (bottom).

\section{CONCLUSION}

Through simulations in Field II mono-static synthetic aperture focusing of pulse-echo data from a rotating concave element has been investigated. The radial and angular resolution have been extracted using transducer elements with a focal depth of $10 \mathrm{~mm}, 15 \mathrm{~mm}$, and $20 \mathrm{~mm}$. Due to the rotation, the synthesized aperture only experiences a moderate expansion.
This is not sufficient to reduce the extent of the wide point spread function of a single emission. At least not to a level which is competitive to the relatively narrow PSF of the setup with focal depth at $20 \mathrm{~mm}$. The effect of SAF with focal depth at $20 \mathrm{~mm}$ is also negligible due to the small number of LRL added.

The advantage of the SAF is the increase in SNR. For the setup with focal depth at $20 \mathrm{~mm}$ the SAF SNR gain is $11 \mathrm{~dB}$. The SNR gain of a setup with a VS at radius $10 \mathrm{~mm}$ or 15 $\mathrm{mm}$ over conventional imaging with a VS at $20 \mathrm{~mm}$ is also $11 \mathrm{~dB}$. The SNR improvement can be converted directly to an increase in penetration or an increase in emitted frequency yielding a higher resolution.

\section{ACKNOWLEDGMENT}

This work was supported by grant 9700883, 9700563 and 26-04-0024 from the Danish Science Foundation and by B-K Medical, Denmark.

\section{REFERENCES}

[1] J. T. Ylitalo and H. Ermert, "Ultrasound synthetic aperture imaging: monostatic approach," IEEE Trans. Ultrason., Ferroelec., Freq. Contr. 41, pp. 333-339, 1994.

[2] M. Karaman, P. C. Li, and M. O’Donnell, "Synthetic aperture imaging for small scale systems," IEEE Trans. Ultrason., Ferroelec., Freq. Contr. 42, pp. 429-442, 1995.

[3] G. R. Lockwood, J. R. Talman, and S. S. Brunke, "Real-time 3-D ultrasound imaging using sparse synthetic aperture beamforming," IEEE Trans. Ultrason., Ferroelec., Freq. Contr. 45, pp. 980-988, 1998.

[4] C. R. Hazard and G. R. Lockwood, "Theoretical assessment of a synthetic aperture beamformer for real-time 3-D imaging," IEEE Trans. Ultrason., Ferroelec., Freq. Contr. 46, pp. 972-980, 1999.

[5] M. O'Donnell and L. J. Thomas, "Efficient synthetic aperture imaging from a circular aperture with possible application to catheter-based imaging," IEEE Trans. Ultrason., Ferroelec., Freq. Contr. 39, pp. 366380, 1992.

[6] C. Passmann and H. Ermert, "A 100-MHz ultrasound imaging system for dermatologic and ophthalmologic diagnostics," IEEE Trans. Ultrason., Ferroelec., Freq. Contr. 43, pp. 545-552, 1996.

[7] C. H. Frazier and W. D. O'Brien, "Synthetic aperture techniques with a virtual source element," IEEE Trans. Ultrason., Ferroelec., Freq. Contr. 45, pp. 196-207, 1998.

[8] S. I. Nikolov and J. A. Jensen, "Virtual ultrasound sources in highresolution ultrasound imaging," in Proc. SPIE - Progress in biomedical optics and imaging, 3, pp. 395-405, 2002.

[9] S. I. Nikolov and J. A. Jensen, "3D synthetic aperture imaging using a virtual source element in the elevation plane," in Proc. IEEE Ultrason. Symp., 2, pp. 1743-1747, 2000.

[10] M. H. Bae and M. K. Jeong, "A study of synthetic-aperture imaging with virtual source elements in B-mode ultrasound imaging systems," in IEEE Trans. Ultrason., Ferroelec., Freq. Contr., 47, pp. 1510-1519, 2000.

[11] J. A. Jensen and N. B. Svendsen, "Calculation of pressure fields from arbitrarily shaped, apodized, and excited ultrasound transducers," IEEE Trans. Ultrason., Ferroelec., Freq. Contr. 39, pp. 262-267, 1992.

[12] J. A. Jensen, "Field: A program for simulating ultrasound systems," Med. Biol. Eng. Comp. 10th Nordic-Baltic Conference on Biomedical Imaging, Vol. 4, Supplement 1, Part 1, pp. 351-353, $1996 \mathrm{~b}$.

[13] J. Kortbek, S. I. Nikolov, and J. A. Jensen, "Effective and versatile software beamformation toolbox," in Proc. SPIE - Medical Imaging Ultrasonic Imaging and Signal Processsing, pp. 651319,1-10, 2007.

[14] N. Oddershede and J. A. Jensen, "Effects influencing focusing in synthetic aperture vector flow imaging," IEEE Trans. Ultrason., Ferroelec., Freq. Contr. 54, pp. 1811-1825, September 2007. 